\title{
Pacific
}

Journal of

Mathematics

\section{CONDITIONAL WIENER INTEGRALS. II}

Chull Park and David Lee Skoug 


\title{
CONDITIONAL WIENER INTEGRALS II
}

\author{
Chull Park and David Skoug
}

In this paper we establish various results involving conditional Wiener integrals, $E(F \mid X)$, for very general conditioning functions $X$. Most related results in the literature, including the case when the conditioning function $X$ is vector-valued, then follow as corollaries of this more general theory. A simple formula is given for converting these generalized conditional Wiener integrals into ordinary Wiener integrals and then this formula is used to evaluate $E(F \mid X)$ for various classes of functionals $F$. Finally these results are used to obtain a generalized conditional form of the Cameron-Martin translation theorem.

1. Introduction. Let $\left(C[0, T], \mathcal{F}^{*}, m_{w}\right)$ denote Wiener space, where $C[0, T]$ is the space of all continuous functions $x$ on $[0, T]$ vanishing at the origin. Let $F(x)$ be a Wiener integrable function on $C[0, T]$ (i.e., $E[|F(x)|]<\infty$ ) and let $X(x)$ be a Wiener measurable function on $C[0, T]$. In [13], Yeh introduced the concept of conditional Wiener integrals. He defined the conditional Wiener integral of $F$ given $X$ as a function on the value space of $X$ and derived a Fourier transform inversion formula for computing conditional Wiener integrals. Using this formula for the case $X(x)=x(T)$, Yeh $[\mathbf{1 3}, \mathbf{1 4}]$ obtained some very useful results including a Kac-Feynman integral equation and a conditional CameronMartin translation theorem.

In [4], for certain functions $F$, Chang and Chang, using Yeh's inversion formula, evaluated the conditional Wiener integral of $F$ given $X(x)=\left(x\left(t_{1}\right), \ldots, x\left(t_{n}\right)\right)$ where $0<t_{1}<t_{2}<\ldots<t_{n}=T$. In [8], the current authors obtained a very simple formula for the conditional Wiener integral of $F$ given $X(x)=\left(x\left(t_{1}\right), \ldots, x\left(t_{n}\right)\right)$. In particular we expressed the conditional Wiener integral directly in terms of an ordinary (i.e., nonconditional) Wiener integral. Using this formula it was relatively simple to generalize the Kac-Feynman formula and to obtain a conditional Cameron-Martin translation theorem involving vector-valued conditioning functions. 
In this paper we consider much more general conditioning functions. In particular they need not depend upon the values of $x$ at only finitely many points in $(0, T]$. A major thrust of this paper is to develop a useful formula to convert these generalized conditional Wiener integrals into ordinary (i.e., nonconditional) Wiener integrals and then to obtain the corresponding Cameron-Martin translation theorem for these generalized conditional Wiener integrals. We also use this simple formula to compute the generalized conditional Wiener integral for various functions $F(x)$ on $C[0, T]$. Most of the results in $[4,8,13$, and 14] then follow as special cases of the results obtained in this paper.

2. Preliminaries and definitions. Let $\mathcal{H}$ be an infinite dimensional subspace of $L_{2}[0, T]$ with a complete orthonormal basis $\left\{\alpha_{j}\right\}$. Then the corresponding stochastic integrals

$$
\gamma_{j}(x)=\int_{0}^{T} \alpha_{j}(t) d x(t), j=1,2, \ldots
$$

form a set of independent standart Gaussian variables on $C[0, T]$ with

$$
E\left[x(t) \gamma_{j}(x)\right]=\int_{0}^{t} \alpha_{j}(s) d s \equiv \beta_{j}(t) .
$$

For each $n \in \mathbb{N}$ let $\mathcal{H}_{n}$ be the subspace of $\mathcal{H}$ spanned by $\left\{\alpha_{1}, \ldots, \alpha_{n}\right\}$, and let $X_{n}: C[0, T] \rightarrow \mathbb{R}^{n}$ and $X_{\infty}: C[0, T] \rightarrow \mathbb{R}^{n}$ be defined by

$$
X_{n}(x)=\left(\gamma_{1}(x), \ldots, \gamma_{n}(x)\right), X_{\infty}(x)=\left(\gamma_{1}(x), \gamma_{2}(x), \ldots\right)
$$

If $\mathcal{B}^{n}$ denotes the $\sigma$-algebra of Borel sets in $\mathbb{R}^{n}$, then a set of the type

$$
I=\left\{x \in C[0, T]: X_{n}(x) \in B\right\} \equiv X_{n}^{-1}(B), B \in \mathcal{B}^{n}
$$

is called a quasi-Wiener interval (or a Borel cylinder). It is well known that

$$
m_{w}(I)=\int_{B} K_{n}(\vec{\xi}) d \vec{\xi}
$$

where

$$
K_{n}(\vec{\xi})=(2 \pi)^{-\frac{n}{2}} \exp \left\{-\frac{1}{2} \sum_{j=1}^{n} \xi_{j}^{2}\right\}
$$


Let $\mathcal{F}_{n}$ be the $\sigma$-algebra formed by the sets $\left\{X_{n}^{-1}(B): B \in \mathcal{B}^{n}\right\}$, and let $\mathcal{F}$ be the $\sigma$-algebra generated by $\cup_{n=1}^{\infty} \mathcal{F}_{n}$. Then, by the definition of conditional expectations (see Doob [5], Tucker [10] and Yeh [12]) for each $F \in L_{1}\left(C[0, T], m_{w}\right)$,

$$
\begin{aligned}
\mu(B) & \equiv \int_{X_{n}^{-1}(B)} F(x) m_{w}(d x)=\int_{X_{n}^{-1}(B)} E\left(F \mid \mathcal{F}_{n}\right) m_{w}(d x) \\
& =\int_{B} E\left(F(x) \mid X_{n}(x)=\vec{\xi}\right) P_{X_{n}}(d \vec{\xi}) \\
& =\int_{B} E\left(F(x) \mid \gamma_{j}(x)=\xi_{j}, j=1, \ldots, n\right) P_{X_{n}}(d \vec{\xi}), B \in \mathcal{B}^{n}
\end{aligned}
$$

where $P_{X_{n}}(B)=m_{w}\left(X_{n}^{-1}(B)\right)$, and $E\left(F(x) \mid X_{n}(x)=\vec{\xi}\right)$ is a Lebesgue measurable function for $\vec{\xi}$ which is unique up to null sets in $\mathbb{R}^{n}$.

Since $\left\{\mathcal{F}_{n}\right\}$ is an increasing sequence of $\sigma$-algebras of Weiner measurable sets, for $F \in L_{1}\left(C[0, T], m_{w}\right),\left\{E\left(F \mid \mathcal{F}_{n}\right)\right\}$ is a martingale sequence. Thus, $E\left|E\left(F \mid \mathcal{F}_{n}\right)\right| \leq E|F|$ for every $n$, and so by the martingale convergence theorem, $\lim E\left(F \mid \mathcal{F}_{n}\right)=E(F \mid \mathcal{F})$ almost surely and for each $A \in \cup_{n=1}^{\infty} \mathcal{F}_{n}$,

$$
\int_{A} E(F(x) \mid \mathcal{F}) m_{w}(d x)=\lim \int_{A} E\left(F(x) \mid \mathcal{F}_{n}\right) m_{w}(d x) .
$$

From this and (2.6), it follows that for every $B \in \cup_{n=1}^{\infty} \mathcal{B}^{n}$,

$$
\begin{aligned}
& \int_{B} E\left(F(x) \mid \gamma_{j}(x)=\xi_{\jmath}, j=1,2, \ldots\right) P_{X_{\infty}}(d \vec{\xi}) \\
& =\lim \int_{B} E\left(F(x) \mid \gamma_{j}(x)=\xi_{j}, j=1, \ldots, n\right) P_{X_{n}}(d \vec{\xi}),
\end{aligned}
$$

where

$$
\begin{aligned}
P_{X_{n}}(d \vec{\xi}) & =\prod_{j=1}^{n}\left\{(2 \pi)^{-\frac{1}{2}} \exp \left(-\xi_{j}^{2} / 2\right) d \xi_{j}\right\} \\
P_{X_{\infty}}(d \vec{\xi}) & =\prod_{j=1}^{\infty}\left\{(2 \pi)^{-\frac{1}{2}} \exp \left(-\xi_{j}^{2} / 2\right) d \xi_{j}\right\}
\end{aligned}
$$

In (2.8) we used the convention that if $B \in \mathcal{B}^{n}$, then $B \in \mathcal{B}^{n+k}$ by identifying $B$ and $B \times \mathbb{R}^{k}$ in $\mathcal{B}^{n+k}$ for $k=1,2, \ldots$ Thus if 
$B \in \cup_{n=1}^{\infty} \mathcal{B}^{n}$, then there exists $N \in \mathbb{N}$ such that $B \in \mathcal{B}^{n}$ for all $n \geq N$, and hence by the martingale property

$$
\begin{aligned}
& \int_{B} E\left(F(x) \mid \gamma_{j}(x)=\xi_{j}, j=1,2, \ldots\right) P_{X_{\infty}}(d \vec{\xi}) \\
& =\int_{B} E\left(F(x) \mid \gamma_{j}(x)=\xi_{j}, j=1, \ldots, n\right) P_{X_{n}}(d \vec{\xi}), \text { for all } n \geq N,
\end{aligned}
$$

from which (2.8) follows.

In the next section we develop quite simple formulas for converting the generalized conditional Wiener integrals of the types $E\left(F(x) \mid X_{n}(x)=\vec{\xi}\right)=E\left(F(x) \mid \gamma_{j}(x)=\xi_{j}, \quad j=1, \ldots, n\right)$ and $E\left(F(x) \mid \gamma_{j}(x)=\xi_{j}, j=1,2, \ldots\right)$ into ordinary Weiner integrals which can often be computed explicitly. It then turns out that all the conditional Weiner integrals that occur in $[4,8,13$, and 14] are special cases of conditional expectations given in this paper.

3. Useful formulas for conditional Wiener integrals. Let $\mathcal{H},\left\{\alpha_{j}\right\}, \mathcal{H}_{n}$ and $\left\{\gamma_{j}(x)\right\}$ be as in Section 2. Define projection maps $\mathcal{P}$ and $\mathcal{P}_{n}$ from $L_{2}[0, T]$ into $\mathcal{H}$ and $\mathcal{H}_{n}$, respectively, by

$$
\begin{gathered}
\mathcal{P} h(t)=\sum_{j=1}^{\infty}\left(h, \alpha_{j}\right) \alpha_{j}(t), \\
\mathcal{P}_{n} h(t)=\sum_{j=1}^{n}\left(h, \alpha_{j}\right) \alpha_{j}(t) .
\end{gathered}
$$

For $x \in C[0, T]$ and $\vec{\xi}=\left(\xi_{1}, \xi_{2}, \ldots\right)$, let

$$
\begin{aligned}
& x_{n}(t)=\int_{0}^{T} \mathcal{P}_{n} I_{[0, t]}(s) d x(s)=\sum_{j=1}^{n} \gamma_{j}(x) \int_{0}^{t} \alpha_{\jmath}(s) d s, \\
& \vec{\xi}_{n}(t)=\sum_{j=1}^{n} \xi_{j}\left(\alpha_{j}, I_{[0, t]}\right),
\end{aligned}
$$

where $I_{[0, t]}$ is the indicator function of the interval $[0, t]$. Similarly, define

$$
\begin{aligned}
& x_{\infty}(t)=\int_{0}^{T} \mathcal{P} I_{[0, t]}(s) d x(s)=\sum_{j=1}^{\infty} \gamma_{j}(x) \int_{0}^{t} \alpha_{j}(s) d s, \\
& \vec{\xi}_{\infty}(t)=\sum_{j=1}^{\infty} \xi_{j}\left(\alpha_{j}, I_{[0, t]}\right) .
\end{aligned}
$$


We note here that since $\left\{\gamma_{j}(x)\right\}$ is a sequence of i.i.d. standard Gaussian random variables, the series $x_{\infty}(t)$ converges $m_{w}$-a.e. $x$ (see Shepp [9, p.324]). Since $\vec{\xi}_{\infty}(t)$ is the evaluation of the random variable $x_{\infty}(t)$ for $\gamma_{j}(x)=\xi_{j}, j=1,2, \ldots, \vec{\xi}_{\infty}(t)$ converges $P_{x_{\infty}}$ - a.e. $\vec{\xi}$.

Our first theorem plays a key role throughout this paper.

ThEOREM 1. If $\{x(t), 0 \leq t \leq T\}$ is the standart Wiener process, then the processes $\left\{x(t)-x_{\infty}(t), 0 \leq t \leq T\right\}$ and $\gamma_{j}(x)$ are (stochastically) independent for $j=1,2, \ldots$ Also, $\{x(t)-$ $\left.x_{n}(t), 0 \leq t \leq T\right\}$ and $\gamma_{j}(x)$ are independent for $j=1, \ldots, n$.

Proof. For each $j$, using (2.2), (3.1) and (3.2)

$$
E\left[\gamma_{j}(x)\left\{x(t)-x_{\infty}(t)\right\}\right]=\int_{0}^{t} \alpha_{j}(s) d s-\sum_{i=1}^{\infty} \delta_{i j} \int_{0}^{t} \alpha_{j}(s) d s=0 .
$$

Since both $\gamma_{j}(x)$ and $x(t)-x_{\infty}(t)$ are Gaussian and uncorrelated, it follows that they are independent. The second claim follows in similar manner.

Corollary 1. The processes $\left\{x(t)-x_{\infty}(t), 0 \leq t \leq T\right\}$ and $\left\{x_{\infty}(t), 0 \leq t \leq T\right\}$ are independent, and so are $\left\{x(t)-x_{n}(t), 0 \leq\right.$ $t \leq T\}$ and $\left\{x_{n}(t), 0 \leq t \leq T\right\}$.

The following theorem is one of our main results.

Theorem 2. Let $F \in L_{1}\left(C[0, T], m_{w}\right)$. Then

$$
\begin{aligned}
& E\left[F(x) \mid \gamma_{j}(x)=\xi_{j}, j=1,2, \ldots\right]=E\left[F\left(x-x_{\infty}+\vec{\xi}_{\infty}\right)\right], \text { and } \\
& E\left[F(x) \mid \gamma_{j}(x)=\xi_{j}, j=1, \ldots, n\right]=E\left[F\left(x-x_{n}+\vec{\xi}_{n}\right)\right] .
\end{aligned}
$$

Proof. Since $x-x_{\infty}$ and $x_{\infty}$ are independent processes, and $\gamma_{j}(x)$ and $x-x_{\infty}$ are independent by Theorem 1 , we may write

$$
\begin{aligned}
& E\left[F(x) \mid \gamma_{j}(x)=\xi_{j}, j=1,2, \ldots\right] \\
& =E\left[F\left(\left(x-x_{\infty}\right)+x_{\infty}\right) \mid \gamma_{j}(x)=\xi_{j}, j=1,2, \ldots\right] \\
& =E_{y}\left\{E_{x}\left[F\left(\left(y-y_{\infty}\right)+x_{\infty}\right) \mid \gamma_{j}(x)=\xi_{j}, j=1,2, \ldots\right]\right\}
\end{aligned}
$$


where $y$ is a standart Wiener process independent of $x$. Thus, we have

$$
\begin{aligned}
& E\left[F(x) \mid \gamma_{j}(x)=\xi_{j}, j=1,2, \ldots\right] \\
& =E_{y}\left\{F\left(\left(y-y_{\infty}\right)+\vec{\xi}_{\infty}\right)\right\}=E\left[F\left(x-x_{\infty}+\vec{\xi}_{\infty}\right)\right]
\end{aligned}
$$

as $x_{\infty}=\vec{\xi}_{\infty}$ under the condition $\gamma_{j}=\xi_{j}, j=1,2, \ldots$ The second formula of (3.4) follows by the same reasoning.

Corollary 2. Let $F \in L_{1}\left(C[0, T], m_{w}\right)$. If $\mathcal{H}=L_{2}[0, T]$, then $E\left[F(x) \mid \gamma_{j}(x)=\xi_{j}, j=1,2, \ldots\right]=F\left(\vec{\xi}_{\infty}\right)$.

Proof. This follows from (3.4) by the fact that if $\mathcal{H}=L_{2}[0, T]$, then $x(t)=\int_{0}^{T} I_{[0, t]}(s) d s(s)=\sum_{j=1}^{\infty}\left(\alpha_{j}, I_{[0, t]}\right) \gamma_{j}(x)=x_{\infty}(t)$ for $m_{w}$ a.e. $x$.

Corollary 3. Let $F \in L_{1}\left(C[0, T], m_{w}\right)$. Then for every $B \in \mathcal{B}^{n}$,

$$
\int_{X_{n}^{-1}(B)} F(x) m_{w}(d x)=\int_{B} E\left[F\left(x-x_{n}+\vec{\xi}_{n}\right) P_{X_{n}}(d \vec{\xi}) .\right.
$$

The above corollary is a simple consequence of the second formula in (3.4). In addition Theorem 4 on page 114 of [2] is a special case of Corollary 3 above with $B=\mathbb{R}^{n}$.

\section{REMARKS.}

(i) For each partition $\tau \equiv \tau_{n}=\left\{t_{1}, \ldots, t_{n}\right\}$ of $[0, T]$ with $0=t_{0}<$ $t_{1}<\ldots<t_{n}=T$, let $X_{\tau}: C[0, T] \rightarrow \mathbb{R}^{n}$ be defined by $X_{\tau}(x)=$ $\left(x\left(t_{1}\right), \ldots, x\left(t_{n}\right)\right)$. In [8], the current authors considered vectorvalued conditional Wiener integrals of the type $E\left(F(x) \mid X_{\tau}(x)=\vec{\xi}\right)$ for $F \in L_{1}\left(C[0, T], m_{w}\right)$. We note that these can be rewritten in the form

$$
\text { (3.5) } \begin{aligned}
& E\left(F(x) \mid X_{\tau}(x)=\vec{\xi}\right)=E\left(F(x) \mid x\left(t_{j}\right)=\xi_{j}, j=1, \ldots, n\right) \\
& =E\left(F(x) \mid x\left(t_{j}\right)-x\left(t_{j-1}\right)=\xi_{j}-\xi_{j-1}, j=1, \ldots, n\right) \\
& =E\left(F(x) \mid \int_{0}^{T} \alpha_{j}(t) d x(t)=\frac{\xi_{j}-\xi_{j-1}}{\sqrt{t_{j}-t_{j-1}}}, j=1, \ldots, n\right)
\end{aligned}
$$

where $\xi_{0}=t_{0}=0$ and

$$
\alpha_{j}(t)=I_{\left[t_{j-1}, t_{j}\right]}(t) / \sqrt{t_{j}-t_{j-1}}, j=1, \ldots, n .
$$


Since $\left\{\alpha_{1}(t), \ldots, \alpha_{n}(t)\right\}$ is obviously an orthonormal set of functions in $L_{2}[0, T]$, the vector-valued conditional Wiener integral $E\left(F(x) \mid X_{\tau}(x)=\vec{\xi}\right)$ is a special case of the general conditional Wiener integrals of the type $E\left(F(x) \mid X_{n}(x)=\vec{\xi}\right)$ considered in this paper. Thus the conditional Wiener integrals that occur in [4], [8], [13] and [14] are all special cases of those of the type $E\left(F(x) \mid X_{n}(x)=\vec{\xi}\right)$ for appropriate $n$ and $\alpha_{1}, \ldots, \alpha_{n}$.

It is also interesting to note that for each $x \in C[0, T]$ the polygonal function $[x]$ defined by

$$
\begin{gathered}
{[x](t)=x\left(t_{j-1}\right)+\frac{t-t_{j-1}}{t_{j}-t_{j-1}}\left(x\left(t_{j}\right)-x\left(t_{j-1}\right)\right),} \\
t_{j-1} \leq t \leq t_{j}, j=1, \ldots, n
\end{gathered}
$$

has another representation, namely

$$
[x](t)=x_{n}(t), 0 \leq t \leq T
$$

where the $\alpha_{j}$ 's are given by (3.6) and $x_{n}(t)$ is given by (3.2). The formula in [8], p.385, corresponding to (3.4) above is

$$
E\left(F(x) \mid X_{\tau}(x)=\vec{\xi}\right)=E[F(x-[x]+[\vec{\xi}])]
$$

where for $\vec{\xi} \in \mathbb{R}^{n},[\vec{\xi}](t)$ is the polygonal function

$$
\begin{aligned}
{[\vec{\xi}](t) } & =\xi_{j-1}+\frac{t-t_{j-1}}{t_{j}-t_{j-1}}\left(\xi_{j}-\xi_{j-1}\right), t_{j-1} \leq t \leq t_{j}, j=1, \ldots, n \\
& =\vec{\xi}_{n}(t)
\end{aligned}
$$

where the $\alpha_{j}$ 's are given by (3.6) and $\vec{\xi}_{n}(t)$ is given by (3.2).

(ii) Thanks to the referee's suggestions, this paper has gone through a number of improvements. The expressions given by (3.2) and (3.3) were suggested by the referee. This in turn, strengthened Theorems 1 and 2. Another suggestion made by the referee was the possibility of generalizing Theorem 2 to other Gaussian processes. This question is perhaps best handled by using the representation of a Gaussian process using Wiener processes; see [7] and example 3 below. 
We close this section with some examples which illustrate that formulas (3.4) are indeed very useful and easy to apply. In particular, the third example deals with the Ornstein-Uhlenbeck process to show that our formulas can be applied to other useful Gaussian processes.

ExAmple 1. For $x \in C[0, T]$ let $F(x)=\int_{0}^{T} x^{2}(t) d t$. Then using (3.4) we obtain

$$
\begin{aligned}
& E\left[\int_{0}^{T} x^{2}(t) d t \mid X_{\alpha}(x)=\vec{\xi}\right] \\
& \quad=E\left[\int_{0}^{T}\left(x(t)-x_{n}(t)+\vec{\xi}_{n}(t)\right)^{2} d t\right] \\
& =\int_{0}^{T} E\left[\left(x(t)-x_{n}(t)\right)^{2}+\left(\vec{\xi}_{n}(t)\right)^{2}+2 \vec{\xi}_{n}(t)\left(x(t)-x_{n}(t)\right)\right] d t .
\end{aligned}
$$

Since $x-x_{n}$ and $x_{n}$ are independent by Corollary $1, E\left[x_{n}(t)(x(t)-\right.$ $\left.\left.x_{n}(t)\right)\right]=0$, and using (2.2) and the fact that $E[x(s) x(t)]=\min \{s, t\}$, we obtain

$$
E\left[\int_{0}^{T} x^{2}(t) d t \mid X_{n}(x)=\vec{\xi}\right]=\int_{0}^{T}\left\{t+\left(\vec{\xi}_{n}(t)\right)^{2}-\sum_{j=1}^{n} \beta_{j}^{2}(t)\right\} d t .
$$

In particular, if $n=1$ and $\alpha(s)=1 / \sqrt{T}$, we see that

$$
\begin{aligned}
E\left[\int_{0}^{T} x^{2}(t) d t \mid X_{1}(x)=\xi\right] & =E\left[\int_{0}^{T} x^{2}(t) d t \mid x(T)=\xi\right] \\
& =\int_{0}^{T}\left\{t+\frac{\xi^{2} t^{2}}{T^{2}}-\frac{t^{2}}{T}\right\} d t=\frac{T^{2}}{6}+\frac{\xi^{2} T}{3}
\end{aligned}
$$

which agrees with the results in [4], [8] and [13].

ExAmple 2. For $x \in C[0, T]$ let $F(x)=\exp \left\{\int_{0}^{T} x(t) d t\right\}$. Then

$$
\begin{aligned}
E[\exp & \left.\left\{\int_{0}^{T} x(t) d t\right\} \mid X_{n}(x)=\vec{\xi}\right] \\
& =E\left[\exp \left\{\int_{0}^{T}\left(x(t)-x_{n}(t)+\vec{\xi}_{n}(t)\right) d t\right\}\right] \\
& =\exp \left\{\int_{0}^{T} \vec{\xi}_{n}(t) d t\right\} E\left[\exp \left\{\int_{0}^{T}\left(x(t)-x_{n}(t)\right) d t\right\}\right] .
\end{aligned}
$$


In particular, if we choose the complete orthonormal cosine sequence $\alpha_{j}(t)=\sqrt{2 / T} \cos [(j-1 / 2) \pi t / T], j=1,2, \ldots$, on $[0, T]$, then it is well known (see Shepp [9], p.325) that the corresponding $x_{n}(t)$ converges to $x(t)$ uniformly in $t$ with probability one, and for each $u \in C[0, T]$,

$$
\sum_{j=1}^{\infty} \int_{0}^{T}\left\{\int_{0}^{t} \alpha_{j}(s) d s \int_{0}^{T} \alpha_{j}(s) d u(s)\right\} d t=\int_{0}^{T} u(t) d t .
$$

Thus

$$
\lim _{n \rightarrow \infty} E\left[\exp \left\{\int_{0}^{T} x(t) d t\right\} \mid X_{n}(x)=X_{n}(u)\right]=\exp \left\{\int_{0}^{T} u(t) d t\right\}
$$

as expected. Since the orthonormal cosine sequence given above is complete on $[0, T]$, Corollary 2 can be applied to get

$$
\begin{aligned}
E\left[\exp \left\{\int_{0}^{T} x(t) d t\right\} \mid \gamma_{j}(x)=\gamma_{j}(u), j=1,2, \ldots\right] & \\
& =\exp \left\{\int_{0}^{T} u(t) d t\right\}
\end{aligned}
$$

for a.e. $u \in C[0, T]$.

EXAMPLE 3. Consider the Ornstein-Uhlenbeck process $y(t)$ with mean zero and covariance function $R(s, t)=\sigma^{2} \exp \{-\beta|t-s|\}$ where $\beta>0$. If we take $\sigma=\beta=1$ for convenience, then $y(t)$ can be expressed in terms of the standart Wiener process $x(t)$ (see p.414 of $[7])$,

$$
y(t)=e^{-t} x\left(e^{2 t}\right), 0 \leq t \leq T .
$$

Suppose $F(y)$ is an integrable function of $y$. Let $\tau=\left\{0=t_{0}, t_{1}, \ldots\right.$, $\left.t_{n}=T\right\}$ be a partition of $[0, T]$. Then, the conditional expectation

$$
E\left[F(y) \mid y\left(t_{j}\right)=\xi_{j}, j=0,1, \ldots, n\right]
$$

can be expressed as a non-conditional expectation by utilizing (3.7). Since $e^{t} y(t)=x\left(e^{2 t}\right)$ and $x(\cdot)$ has independent increments, we write

$$
\begin{aligned}
& E\left[F(y) \mid y\left(t_{j}\right)=\xi_{j}, j=0,1, \ldots, n\right] \\
& =E\left[F(y) \mid e^{t_{j}} y\left(t_{j}\right)-e^{t_{j-1}} y\left(t_{j-1}\right)=e^{t_{j}} \xi_{j}-e^{t_{j-1}} \xi_{j-1}, j=0, \ldots, n\right]
\end{aligned}
$$


where $y\left(t_{-1}\right)=\xi_{-1}=0$.

Define $\left(y_{n}\right)(t)$ by

$$
\left(y_{n}\right)(t)=e^{-t}\left[e^{t_{j-1}} y\left(t_{j-1}\right)+\frac{e^{2 t}-e^{2 t_{j-1}}}{e^{2 t_{j}}-e^{2 t_{j-1}}}\left(e^{t_{j}} y\left(t_{j}\right)-e^{t_{j-1}} y\left(t_{j-1}\right)\right)\right]
$$

for $t_{j-1} \leq t \leq t_{j}, j=1, \ldots, n$.

Similarly, define $\left(\vec{\xi}_{n}\right)(t)$ by

$$
\left(\vec{\xi}_{n}\right)(t)=e^{-t}\left[e^{t_{j-1}} \xi_{j-1}+\frac{e^{2 t}-e^{2 t_{j-1}}}{e^{2 t_{j}}-e^{2 t_{j-1}}}\left(e^{t_{j}} \xi_{j}-e^{t_{j-1}} \xi_{j-1}\right)\right]
$$

for $t_{j-1} \leq t \leq t_{j}, j=1, \ldots, n$.

Then, $\left(y_{n}\right)\left(t_{j}\right)=y\left(t_{j}\right)$ and $\left(\vec{\xi}_{n}\right)\left(t_{j}\right)=\xi_{j}$ at each $t_{j} \in \tau$. Furthermore, $\left(y_{n}\right)$ and $y-\left(y_{n}\right)$ are independent processes as one can easily check using the covariance function of $y$. Thus, we conclude that

$$
E\left[F(y) \mid y\left(t_{j}\right)=\xi_{j}, j=0,1, \ldots, n\right]=E\left[F\left(y-\left(y_{n}\right)+\left(\vec{\xi}_{n}\right)\right)\right] .
$$

\section{Conditional expectation of functions involving stochas-} tic integrals. Using the same notation as in section 3 above, for $h \in L_{2}[0, T]$ let

$$
\begin{aligned}
& h_{(n)}(t)=\mathcal{P}_{n} h(t)=\sum_{j=1}^{n}\left(h, \alpha_{j}\right) \alpha_{j}(t) \text { and } \\
& h_{(\infty)}(t)=\mathcal{P}_{\infty} h(t)=\sum_{j=1}^{\infty}\left(h, \alpha_{j}\right) \alpha_{j}(t)
\end{aligned}
$$

Then, we have the following:

Lemma 1. Let $h \in L_{2}[0, T]$. Then

$$
\int_{0}^{T} h(t) h_{(n)}(t) d t=\int_{0}^{T} h_{(n)}^{2}(t) d t=\left\|h_{(n)}\right\|^{2}=\sum_{j=1}^{n}\left(h, \alpha_{j}\right)^{2}
$$

and

$$
\left\|h-h_{(n)}\right\|^{2}=\|h\|^{2}-\left\|h_{(n)}\right\|^{2} .
$$

Obviously, the above formulas hold when $n=\infty$, and $\| h-$ $h_{(\infty)} \|=0$ if $\mathcal{H}=L_{2}[0, T]$. 
Our next theorem gives an interesting relationship involving $h$, $h_{(n)}, x$ and $x_{n}$ that is very useful in computing conditional and ordinary expectations of functions involving the stochastic integral $\int_{0}^{T} h(t) d x_{n}(t)$.

THEOREM 3. Let $h \in L_{2}[0, T]$. Then for each $x \in C[0, T]$

$$
\int_{0}^{T} h(t) d x_{n}(t)=\int_{0}^{T} h_{(n)}(t) d x(t)=\int_{0}^{T} h_{(n)}(t) d x_{n}(t)
$$

The formula also holds for $n=\infty$ if we consider $\int_{0}^{T} h(t) d x_{\infty}(t)=$ $\sum_{j=1}^{\infty} \gamma_{j}(x)\left(h, \alpha_{j}\right)$

Proof. Using 3.1, 3.2, 4.1 and the fact that the $\alpha_{j}$ 's are orthonormal, it is quite easy to show that for each $x \in C[0, T]$, each of the stochastic integrals in 4.4 equals the expression

$$
\sum_{j=1}^{n}\left(h, \alpha_{j}\right) \int_{0}^{T} \alpha_{j}(t) d x(t)
$$

Corollary 4. Let $h \in L_{2}[0, T]$. Then

$$
E\left[\exp \left\{-\int_{0}^{T} h(t) d x_{n}(t)\right\}\right]=\exp \left\{\frac{1}{2}\left\|h_{(n)}\right\|^{2}\right\} .
$$

Proof. By 4.4 and a well known Wiener integration formula

$$
\begin{aligned}
E[\exp & \left.\left\{-\int_{0}^{T} h(t) d x_{n}(t)\right\}\right] \\
& =E\left[\exp \left\{-\int_{0}^{T} h_{(n)}(t) d x(t)\right\}\right] \\
& =(2 \pi)^{-1 / 2} \int_{-\infty}^{\infty} \exp \left\{-\left\|h_{(n)}\right\| u\right\} \exp \left\{-\frac{u^{2}}{2}\right\} d u \\
& =\exp \left\{\frac{1}{2}\left\|h_{(n)}\right\|^{2}\right\} .
\end{aligned}
$$


THEOREM 4. Let $h \in L_{2}[0, T]$ and assume that

$$
F(x)=f\left[\int_{0}^{T} h(t) d x(t)\right]
$$

is in $L_{1}\left(C[0, T], m_{w}\right)$.

a). If $h$ is a linear combination of $\left\{\alpha_{1}, \ldots, \alpha_{n}\right\}$, say $h(t)=$ $c_{1} \alpha_{1}(t)+\ldots+c_{n} \alpha_{n}(t)$ on $[0, T]$, then

(4.6) $E\left[f\left[\int_{0}^{T} h(t) d x(t)\right] \mid X_{n}(x)=\vec{\xi}\right]=f\left(c_{1} \xi_{1}+\ldots+c_{n} \xi_{n}\right)$.

b). If $\left\{h, \alpha_{1}, \ldots, \alpha_{n}\right\}$ is a linearly independent set of functions in $L_{2}[0, T]$, then

$$
\begin{aligned}
E & {\left[f\left[\int_{0}^{T} h(t) d x(t)\right] \mid X_{n}(x)=\vec{\xi}\right] } \\
= & {\left[2 \pi\left(\|h\|^{2}-\left\|h_{(n)}\right\|^{2}\right)\right]^{-1 / 2} } \\
& \cdot \int_{-\infty}^{\infty} f(u) \exp \left\{-\frac{\left(u-\int_{0}^{T} h(t) d \vec{\xi}_{n}(t)\right)^{2}}{2\left\|h-h_{(n)}\right\|^{2}}\right\} d u
\end{aligned}
$$

Proof. a).In this case $h_{(n)}(t) \equiv h(t)$ and so by $3.4,4.4$ and 3.2 ,

$$
\begin{aligned}
E\left[f\left[\int_{0}^{T} h(t) d x(t)\right] \mid X_{n}(x)=\vec{\xi}\right] \\
\quad=E\left[f\left[\int_{0}^{T} h(t) d\left\{x(t)-x_{n}(t)+\vec{\xi}_{n}(t)\right\}\right]\right] \\
\quad=E\left[f\left[\int_{0}^{T}\left(h(t)-h_{(n)}(t)\right) d x(t)+\int_{0}^{T} h(t) d \vec{\xi}_{n}(t)\right]\right] \\
\quad=E\left[f\left[\int_{0}^{T} h(t) d \vec{\xi}_{n}(t)\right]\right] \\
\quad=f\left[\int_{0}^{T} h(t) d \vec{\xi}_{n}(t)\right] \\
=f\left(c_{1} \xi_{1}+\ldots+c_{n} \xi_{n}\right) .
\end{aligned}
$$


b). In this case we use $3.4,4.4$, and a well known Wiener integration formula to obtain

$$
\begin{aligned}
E & {\left[f\left[\int_{0}^{T} h(t) d x(t)\right] \mid X_{n}(x)=\vec{\xi}\right] } \\
& =E\left[f\left[\int_{0}^{T} h(t) d\left\{x(t)-x_{n}(t)+\vec{\xi}_{n}(t)\right\}\right]\right] \\
& =E\left[f\left[\int_{0}^{T}\left(h(t)-h_{(n)}(t)\right) d x(t)+\int_{0}^{T} h(t) d \vec{\xi}_{n}(t)\right]\right] \\
& =(2 \pi)^{-1 / 2} \int_{-\infty}^{\infty} f\left(\left\|h-h_{(n)}\right\| u+\int_{0}^{T} h(t) d \vec{\xi}_{n}(t)\right) \exp \left\{-u^{2} / 2\right\} d u
\end{aligned}
$$

In Theorem 4 above the two extreme cases occur when $h \equiv \alpha_{j}$ for some $j$ or when $h$ is orthogonal to all the $\alpha_{j}$ 's.

Corollary 5. Let $h, F$ and $f$ be as in Theorem 4. Then

$$
E\left[f\left[\int_{0}^{T} \alpha_{j}(t) d x(t)\right] \mid X_{n}(x)=\vec{\xi}\right]=f\left(\xi_{j}\right)
$$

while if $\left\{h, \alpha_{1}, \ldots, \alpha_{n}\right\}$ is an orthogonal set of functions in $L_{2}[0, T]$,

$$
\begin{aligned}
E\left[f\left[\int_{0}^{T} h(t) d x(t)\right]\right. & \left.\mid X_{n}(x)=\vec{\xi}\right]=E\left[f\left[\int_{0}^{T} h(t) d x(t)\right]\right] \\
& =\left[2 \pi\|h\|^{2}\right]^{-1 / 2} \int_{-\infty}^{\infty} f(u) \exp \left\{-\frac{u^{2}}{2\|h\|^{2}}\right\} d u .
\end{aligned}
$$

Proceeding as above we obtain the following generalization of formula 4.9.

CoROllaRY 6. If $\left\{\phi_{1}, \ldots, \phi_{m}, \alpha_{1}, \ldots, \alpha_{n}\right\}$ is an orthonormal set of functions in $L_{2}[0, T]$ and if

$$
F(x)=f\left[\int_{0}^{T} \phi_{1}(t) d x(t), \ldots, \int_{0}^{T} \phi_{m}(t) d x(t)\right]
$$


is in $L_{1}\left(C[0, T], m_{w}\right)$, then

$$
\begin{aligned}
E(f & {\left.\left[\int_{0}^{T} \phi_{1}(t) d x(t), \ldots, \int_{0}^{T} \phi_{m}(t) d x(t)\right] \mid X_{n}(x)=\vec{\xi}\right) } \\
& =E\left[f\left[\int_{0}^{T} \phi_{1}(t) d x(t), \ldots, \int_{0}^{T} \phi_{m}(t) d x(t)\right]\right] \\
& =\left[\prod_{j=1}^{m}[2 \pi]^{-1 / 2}\right] \int_{\mathbb{R}^{m}} f\left(u_{1}, \ldots, u_{m}\right) \exp \left\{-\sum_{j=1}^{m} \frac{u_{j}^{2}}{2}\right\} d \vec{u} .
\end{aligned}
$$

Our next corollary follows from the observations that $\int_{0}^{T}(h(t)-$ $\left.h_{(n)}(t)\right) d \vec{\xi}_{n}(t)=0$, and $\left(h-h_{(n)}\right)_{(n)}(t)=0$.

Corollary 7. Let $h, F$ and $f$ be as in Theorem 4. Then

$$
\begin{aligned}
E[f & {\left.\left[\int_{0}^{T} h(t) d\left\{x(t)-x_{n}(t)\right\}\right] \mid X_{n}(x)=\vec{\xi}\right] } \\
& =E\left[f\left[\int_{0}^{T}\left\{h(t)-h_{(n)}(t)\right\} d x(t)\right] \mid X_{n}(x)=\vec{\xi}\right] \\
& =E\left[f\left[\int_{0}^{T}\left\{h(t)-h_{(n)}(t)\right\} d x(t)\right]\right] \\
& =\left[2 \pi\left\|h-h_{(n)}\right\|^{2}\right]^{-1 / 2} \int_{-\infty}^{\infty} f(u) \exp \left\{-\frac{u^{2}}{2\left\|h-h_{(n)}\right\|^{2}}\right\} d u
\end{aligned}
$$

Many interesting examples of conditional Wiener integrals can be obtained as special cases of the following theorem.

Theorem 5. Let $g \in L_{2}[0, T]$. Then

$$
\begin{aligned}
& E\left[\exp \left\{\int_{0}^{T} g(s) x(s) d s\right\} \mid X_{n}(x)=\vec{\xi}\right] \\
& \quad=\exp \left\{\sum_{j=1}^{n} \xi_{j}\left(g, \beta_{j}\right)+\frac{1}{2} \int_{0}^{T}\left[\int_{s}^{T} g(t) d t\right]^{2} d s-\frac{1}{2} \sum_{j=1}^{n}\left(g, \beta_{j}\right)^{2}\right\} .
\end{aligned}
$$

Proof. Using integration by parts it follows that 


$$
\int_{0}^{T} g(s) x(s) d s=\int_{0}^{T}\left[\int_{s}^{T} g(t) d t\right] d x(s)
$$

and that

$$
\int_{0}^{T}\left[\int_{s}^{T} g(t) d t\right] \alpha_{j}(s) d s=\int_{0}^{T} g(s) \beta_{j}(s) d s=\left(g, \beta_{j}\right)
$$

Hence using (3.4) we obtain

$$
\begin{aligned}
& E\left[\exp \left\{\int_{0}^{T} g(s) x(s) d s\right\} \mid X_{n}(x)=\vec{\xi}\right] \\
& =E\left[\exp \left\{\int_{0}^{T}\left[\int_{s}^{T} g(t) d t\right] d\left(x(s)-x_{n}(s)+\vec{\xi}_{n}(s)\right)\right\}\right] \\
& =\exp \left\{\sum_{j=1}^{n} \xi_{i} \int_{0}^{T}\left[\int_{s}^{T} g(t) d t\right] \alpha_{j}(s) d s\right\} \\
& \text {. } E\left[\operatorname { e x p } \left\{\int_{0}^{T}\left[\int_{s}^{T} g(t) d t\right] d x(s)\right.\right. \\
& \left.\left.-\sum_{j=1}^{n} \gamma_{j}(x) \int_{0}^{T}\left[\int_{s}^{T} g(t) d t\right] \alpha_{j}(s) d s\right\}\right] \\
& =\exp \left\{\sum_{j=1}^{n} \xi_{j}\left(g, \beta_{j}\right)\right\} \\
& \cdot E\left[\exp \left\{\int_{0}^{T}\left[\int_{s}^{T} g(t) d t-\sum_{\jmath=1}^{n}\left(g, \beta_{j}\right) \alpha_{\jmath}(s)\right] d x(s)\right\}\right] \\
& =\exp \left\{\sum_{j=1}^{n} \xi_{j}\left(g, \beta_{j}\right)+\frac{1}{2} \int_{0}^{T}\left[\int_{s}^{T} g(t) d t-\sum_{j=1}^{n}\left(g, \beta_{j}\right) \alpha_{j}(s)\right]^{2} d s\right\}
\end{aligned}
$$

from which 4.10 follows.

Corollary 8. Let $g(s) \equiv 1$ and let the $\alpha_{j}$ 's be given by 3.6. 
Then

$$
\begin{aligned}
E[\exp & \left.\left\{\int_{0}^{T} x(s) d s\right\} \mid X_{n}(x)=\vec{\xi}\right] \\
= & \exp \left\{\frac{T^{3}}{6}+\frac{1}{2} \sum_{j=1}^{n}\left(\xi_{j}+\xi_{j-1}\right)\left(t_{j}-t_{j-1}\right)\right. \\
& \left.-\frac{1}{8} \sum_{j=1}^{n}\left(t_{j}-t_{j-1}\right)\left(t_{j}+t_{j-1}\right)^{2}\right\} .
\end{aligned}
$$

COROLlaRY 9. Let $n=1$ and $\alpha_{1}(s) \equiv 1 / \sqrt{T}$. Then

$$
\begin{aligned}
& E\left[\exp \left\{\int_{0}^{T} g(s) x(s) d s\right\} \mid x(T)=\xi\right] \\
& =\exp \left\{\frac{\xi}{T} \int_{0}^{T} t g(t) d t+\frac{1}{2} \int_{0}^{T}\left[\int_{s}^{T} g(t) d t\right]^{2} d s-\frac{1}{2 T}\left[\int_{0}^{T} t g(t) d t\right]^{2}\right\}, \\
& E\left[\exp \left\{\int_{0}^{T} s x(s) d s\right\} \mid x(T)=\xi\right]=\exp \left\{\frac{\xi T^{2}}{3}+\frac{T^{5}}{90}\right\},
\end{aligned}
$$

and

$$
E\left[\exp \left\{\int_{0}^{T} x(s) d s\right\} \mid x(T)=\xi\right]=\exp \left\{\frac{\xi T}{2}+\frac{T^{3}}{24}\right\} .
$$

\section{Translation of generalized conditional Wiener integrals.} The Cameron-Martin Theorem [3], [11] states that if $x_{0}(t)=$ $\int_{0}^{t} h(s) d s$ for all $t \in[0, T]$ with $h \in L_{2}[0, T]$, and if $T_{1}$ is the transformation from $C[0, T]$ into itself defined by

$$
T_{1}(x)=x+x_{0} \text { for } x \in C[0, T],
$$

then for any Wiener integrable function $F$ on $C[0, T]$ and any Wiener measurable set $\Gamma$

$$
\int_{\Gamma} F(y) m_{w}(d y)=\int_{T_{1}^{-1}(\Gamma)} F\left(x+x_{0}\right) J\left(x_{0}, x\right) m_{w}(d x)
$$

where

$$
J\left(x_{0}, x\right)=\exp \left\{-\frac{1}{2}\|h\|^{2}-\int_{0}^{T} h(t) d x(t)\right\} .
$$


In particular, if $\Gamma=C[0, T]$, then 5.1 becomes:

$$
E[F(y)]=E\left[F\left(x+x_{0}\right) J\left(x_{0}, x\right)\right]
$$

In [14], Yeh gives a conditional version of 5.3 which states that

$$
\begin{aligned}
& E[F(y) \mid y(T)=\xi]=E\left[F(y) \mid \int_{0}^{T} d y(t)=\xi\right] \\
= & E\left[F\left(x+x_{0}\right) J\left(x_{0}, x\right) \mid x(T)=\xi-x_{0}(T)\right] \exp \left\{-\frac{x_{0}^{2}(T)}{2 T}+\frac{\xi x_{0}(T)}{T}\right\} .
\end{aligned}
$$

Our next theorem is a generalized conditional version of 5.3.

ThEOREM 6. Let $h \in L_{2}[0, T]$ and let $x_{0}(t)=\int_{0}^{t} h(s) d s$ for $t \in[0, T]$. Let $F \in L_{1}\left(C[0, T], m_{w}\right)$ and let the $\alpha_{j}$ 's be as in Section 2. Then

$$
\begin{gathered}
E\left[F(y) \mid X_{\alpha}(y)=\vec{\xi}\right] \\
=E\left[F\left(x+x_{0}\right) J\left(x_{0}, x\right) \mid X_{n}\left(x+x_{0}\right)=\vec{\xi}\right] \\
\quad \cdot \exp \left\{\int_{0}^{T} h(t) d \vec{\xi}_{n}(t)-\frac{1}{2}\left\|h_{(n)}\right\|^{2}\right\}
\end{gathered}
$$

where $J\left(x_{0}, x\right)$ is given by 5.2 and $h_{(n)}(t)$ is given by 4.1 . The result holds for $n=\infty$ as well.

Proof. By 3.4 we see that

$$
E\left[F(y) \mid X_{n}(y)=\vec{\xi}\right]=E\left[F\left(y-y_{n}+\vec{\xi}_{n}\right)\right]
$$

Using 5.3 and noting that $\left(x+x_{0}\right)_{n}=x_{n}+\left(x_{0}\right)_{n}$, we have

$$
E\left[F\left(y-y_{n}+\vec{\xi}_{n}\right)\right]=E\left[F\left(x+x_{0}-x_{n}-\left(x_{0}\right)_{n}+\vec{\xi}_{n}\right) J\left(x_{0}, x\right)\right] .
$$


Next we rewrite $J\left(x_{0}, x\right)$ in the form

$$
\begin{aligned}
J\left(x_{0}, x\right) & =\exp \left\{-\frac{1}{2}\|h\|^{2}\right\} \\
\cdot & \exp \left\{-\int_{0}^{T} h(t) d\left(x(t)-x_{n}(t)+\vec{\xi}_{n}(t)-\left(x_{0}\right)_{n}(t)\right)\right\} \\
\cdot & \exp \left\{-\int_{0}^{T} h(t) d x_{n}(t)\right\} \\
\cdot & \exp \left\{\int_{0}^{T} h(t) d\left(\vec{\xi}_{n}(t)-\left(x_{0}\right)_{n}(t)\right)\right\} .
\end{aligned}
$$

Using 4.1 we see that

$$
\int_{0}^{T} h(t) d\left(x_{0}\right)_{n}(t)=\int_{0}^{T} h_{(n)}^{2}(t) d t=\left\|h_{(n)}\right\|^{2}
$$

Since $x_{n}(t)$ and $x(t)-x_{n}(t)$ are independent processes on $[0, T]$ by Corollary $1, \exp \left\{-\int_{0}^{T} h(t) d x_{n}(t)\right\}$ and

$$
\begin{aligned}
& F\left(x+x_{0}-\right.\left.x_{n}-\left(x_{0}\right)_{n}+\vec{\xi}_{n}\right) \\
& \cdot \exp \left\{-\int_{0}^{T} h(t) d\left(x(t)-x_{n}(t)+\vec{\xi}_{n}(t)-\left(x_{0}\right)_{n}(t)\right)\right\}
\end{aligned}
$$

are also independent. Thus using 5.7, 4.5 and 5.8,

$$
\begin{aligned}
E & {\left[F\left(x+x_{0}-x_{n}-\left(x_{0}\right)_{n}+\vec{\xi}_{n}\right) J\left(x_{0}, x\right)\right] } \\
& =E\left[F\left(x+x_{0}-x_{n}-\left(x_{0}\right)_{n}+\vec{\xi}_{n}\right)\right. \\
& \left.\cdot \exp \left\{-\int_{0}^{T} h(t) d\left(x(t)-x_{n}(t)+\vec{\xi}_{n}(t)-\left(x_{0}\right)_{n}(t)\right)\right\}\right] \\
& \cdot \exp \left\{-\frac{1}{2}\|h\|^{2}+\frac{1}{2}\left\|h_{(n)}\right\|^{2}+\int_{0}^{T} h(t) d \vec{\xi}_{n}(t)-\left\|h_{(n)}\right\|^{2}\right\} .
\end{aligned}
$$


Therefore, by using 5.9 and 3.4 we obtain

$$
\begin{aligned}
E[ & \left.F\left(x+x_{0}-x_{n}-\left(x_{0}\right)_{n}+\vec{\xi}_{n}\right) J\left(x_{0}, x\right)\right] \\
= & E\left(\left[F\left(x+x_{0}\right) \exp \left\{-\int_{0}^{T} h(t) d(x(t))\right\}\right] \mid X_{\alpha}\left(x+x_{0}\right)=\vec{\xi}\right) \\
& \cdot \exp \left\{-\frac{1}{2}\|h\|^{2}+\int_{0}^{T} h(t) d \vec{\xi}_{n}(t)-\frac{1}{2}\left\|h_{(n)}\right\|^{2}\right\} \\
= & E\left(\left[F\left(x+x_{0}\right) J\left(x_{0}, x\right)\right] \mid X_{\alpha}\left(x+x_{0}\right)=\vec{\xi}\right) \\
& \cdot \exp \left\{\int_{0}^{T} h(t) d \vec{\xi}_{n}(t)-\frac{1}{2}\left\|h_{(n)}\right\|^{2}\right\} .
\end{aligned}
$$

This together with 5.6 and 5.5 yields 5.4 . The case $n=\infty$ follows by the martingle convergence theorem.

REMARK. By choosing the $\alpha_{j}$ 's as in 3.6 , we see that Theorem 4 on page 391 of [8] is a Corollary of Theorem 6 above.

Acknowledgement. We dedicate this paper to the memory of Professor Robert H. Cameron (1908 - 1989).

\section{REFERENCES}

[1] L. Breiman, Probability, Addison-Wesley, Reading, Mass. 1968.

[2] R.H. Cameron, A "Simpsons Rule" for the numerical evaluation of Wiener's integrals in function space, Duke Math. J., 18 (1951), 111-130.

[3] R.H. Cameron and W.T. Martin, Transformation of Wiener integrals under translations, Ann. Math., 45 (1944), 386-396.

[4] K.S. Chang and J.S. Chang, Evaluation of some conditional Wiener integrals, Bull. Korean Math. Soc., 21 (1984), 99-106.

[5] J.L. Doob, Stochastic processes, Wiley, New York, 1953.

[6] M. Kac, Probability and Related Topics in Physical Sciences, Interscience Publishers, New York, 1959.

[7] C. Park, Representations of Gaussian processes by Wiener processes, $\mathrm{Pa}$ cific J. Math., 94 (1981), 407-415.

[8] C. Park and D.L. Skoug A simple formula for conditional Wiener integrals with applications, Pacific J. Math., 135 (1988), 381-394.

[9] L.A. Shepp Radon-Nikodym derivative of Gaussian measures, Ann. Math. Stat., 37 (1966), 321-354.

[10] H.G. Tucker, A graduate course in probability, Academic Press, New York, 1967. 
[11] J. Yeh, Stochastic Processes and the Wiener Integral, Marcel Dekker, New York, 1973.

[12] - Stochastic processes and the Weiner integral, Marcel Dekker, New York, 1973.

[13] Inversion of conditional Wiener integrals, Pacific J. Math., 59 (1975), 623-638.

[14] - Transformation of conditional Wiener integrals under translation and the CAmeron-MArtin translation theorem, Tôhoku Math. Journ., 30 (1978), 505-515.

Received September 7, 1991 and in revised form November 5, 1992.

MIAMI UNIVERSITY

OXFORD, OH 45056-1641

E-mail address: cpark@miavx1.acs.muohio.edu

AND

UNIVERSITY OF NEBRASKA

LINCOLN, NE 68588-0323 



\section{PACIFIC JOURNAL OF MATHEMATICS}

Volume $167 \quad$ No. $2 \quad$ February 1995

Existence of shortest directed networks in $\mathbb{R}^{2}$

201

MANUEL AlFARO GARCIA

Hecke characters of singular Drinfel'd modules

215

SUNGHAN BAE

Factorization method for a bimeromorphic morphism

231

Jose PEREZ BLANCO

$L^{p}$ estimates for operators associated to flat curves without the Fourier 243 transform

Anthony Carbery, James Thomas Vance, JR., Stephen

WAINGER, DAVID K. WATSON and JAMES WRIGHT

$S$-integer points on elliptic curves

263

ROBERT HOWARD GROSS and JOSEPH SILVERMAN

On metrics defined by modules

JAMES ALLISTER JENKINS

Conditional Wiener integrals. II

ChUll PARK and DAVID LeE SKOUG

On a Plancherel formula for certain discrete, finitely generated, torsion-free nilpotent groups

CAROLYN PFEFFER JOHNSTON

Desingularizations of some unstable orbit closures

MARK STEPHEN REEDER

Determining multiplicities of half-integral weight newforms

Thomas Richard SHEMANSKE and LyNne WALLING

Generation of integral orthogonal groups over dyadic local fields 